\title{
Comparison for the total expected quality control cost between proposed model and Schmidt-Taylor model
}

\author{
Prof. Dr. Dhwyia Salman Hassan ${ }^{1}$, Dr. Hani Aziz Ameen ${ }^{2}$ and Dr. Waleed A Arhema AL- \\ Elaiwe $^{3}$ \\ ${ }^{1}$ Department of Statistics, College of Administration And Economics, University of Baghdad- \\ Iraq, E-mail: dhwyia.salman@yahoo.com \\ ${ }^{2}$ Technical College- Baghdad- Iraq, E-mail:haniazizameen@yahoo.com \\ ${ }^{3}$ Department of Informative technology, Technical College of management, Baghdad-Iraq, \\ E-mail: drwaleed_2001@yahoo.com
}

\begin{abstract}
In this paper ,we constructed a model for the total expected cost of quality control, which include four components, $\mathrm{A} 1$ (inspection cost) and $\mathrm{A} 2$ represent cost of accepting defective units , and the third component is $A 3$ (cost of rejecting good units) and finally A4 which is the loss due to stopping of production line. This model is modification for the model presented by Schmidt-Taylor [2]. The aim of model is to determine the three parameters $\left(\boldsymbol{n}_{v} \boldsymbol{c}_{v}, \boldsymbol{\tau}\right)$ of single sampling plan, were $(\boldsymbol{n})$ is the sample size,(c) is acceptance number and $(\tau)$ is the time interval between inspections. The proposed model needs the multivariate search technique and enumeration procedure; to solve the expected cost quality model and then obtain the set of parameters that minimize this function the efficiency of proposed model was compared with this due to Schmidt-Taylor model .All notations and derivations are explained.
\end{abstract}

Keyword: Acceptance sampling plan, proposed model for expected total cost of quality control, Multivariate search technique, enumeration procedure application.

\section{The aim of research}

The basic aim of this research is to build linear cost model for total quality control by minimizing the total expected value of cost function which consists of four components $\left(A_{1}, A_{2}, A_{3}, A_{4}\right)$. The percentage of defective is $\left(p_{0}\right)$ in normal conditions of production, and $\left(\boldsymbol{P}_{1}, \boldsymbol{P}_{1}>\boldsymbol{P}_{0}\right)$, when there is reasons which cause the defective in production process. The period time $(\tau)$ between successive inspections is divided into two periods, first is $(t)$ when $(T<t)$ which is the time until the failure in production process happen and this time is random variable distributed exponential with $\left(\right.$ mean $\left.=\frac{1}{\lambda}\right)$ and the second period is $(\tau-t)$ where $(t<T<\tau)$ which is the period since the failure happen and until the production line is returned to its normal position.

The proposed model depends on proportion of defective $p(t)$ in the produced lot when the failure occurs at time $(\mathrm{t})$ from the production of a lot $(\mathrm{N})$.

We proposed the following form (1) to be a general form, can be used for most distribution, in various applications.

The form of $p(t)$ is:
$p(t)=P_{0} p r(T \leq t \mid T<\tau)+p_{1} \operatorname{pr}(t<T<\tau \mid T<\tau) \ldots$ (1)

Form now we assume that $(x)$ is the number of defective in sample (n) and it is r.v. from binomial $(n, p)$ and the time continued until failure happened is r.v. distributed as exponential with rate $\left(\frac{1}{\lambda}\right)$ i.e.

$\begin{array}{rrr}f_{T}(t)=\lambda e^{-\lambda t} & t>0 & \\ 0 & & \text { o.w. }\end{array}$

Notations and Assumption

1) $\boldsymbol{P}_{0}:$ proportion of defective which is accepted

2) $\boldsymbol{P}_{1}::\left(\boldsymbol{P}_{1}:>\boldsymbol{P}_{0}:\right)$ proportion of defective which is rejected

3) N: size of lot

4) n: sample size

5) c: acceptance number

6) Inspection used is of rectifying kind.

7) The decision of accepting sample, implies accepting all the remaining lot(N-n)

8) The decision of rejecting a sample, cause to rejection the lot, and inspected all the quantity $(\mathrm{N}-n)$.

9) The cost of testing and accepting and rejecting and cost due to stopping of 
production line all are in the same units (\$, .....

10) The definition of percentage of defective due to Schmidt-Taylor (1973), which we used for modified model is $p(t)=\frac{p_{0}(t)+p_{1}(\tau-t)}{\tau}$

Which is especial case from proposed equation number (1), were if we solve equation (1) by using $\boldsymbol{T} \sim \boldsymbol{u}(0,1)$ we obtain equation (2)

$\theta_{j}=\theta_{1} \sum_{x=0}^{j-1}\left[p\left(A \mid f_{b}\right)-\theta_{1}\right]^{X}$

$\theta_{1}=\sum_{x=0}^{c} \int_{0}^{\pi} f_{T}(t) P_{X}\left(x \mid n_{s} p\right) d t$

We may re-write in the following form

Since:-

$\theta_{1}=p(d) p\left(A \mid f_{d}\right) \ldots(3)$

$\theta_{2}=\theta_{1} p\left(A \mid f_{b}\right)+\left(1-\theta_{1}\right) p(d) p\left(A \mid f_{d}\right)$

$=\theta_{1} p\left(A \mid f_{b}\right)+\left(1-\theta_{1}\right) \theta_{1}$

$=\theta_{1}\left[p\left(A \mid f_{b}\right)+\left(1-\theta_{1}\right)\right] \ldots(4)$

Similarly:-

$\theta_{3}=\theta_{2} p\left(A \mid f_{b}\right)+\left(1-\theta_{2}\right) \theta_{1}$

$=$

$\bar{\theta}_{1}\left[p\left(A \mid f_{b}\right)+\left(1-\theta_{1}\right)\right] p\left(A \mid f_{b}\right)+\theta_{1}-\theta_{1}^{2}\left[p\left(A \mid f_{b}\right)+\left(1-\theta_{1}\right)\right]$

$=\theta_{1}\left[\left\{p\left(A \mid f_{b}\right)-\theta_{1}\right\}^{2}+\left\{p\left(A \mid f_{b}\right)-\theta_{1}\right\}+1\right] \ldots(5)$

Then the probability that the production line is in case of failure after jth inspection is:-

$\theta_{j}=\theta_{1} \sum_{x=0}^{j-1}\left[p\left(A \mid f_{b}\right)-\theta_{1}\right]^{X}$

Then

$B=\lim _{j=x} \theta_{j}=\frac{\theta_{1}}{1-p\left(A \mid f_{b}\right)+\theta_{1}} \ldots(7)$

Where

$\theta_{1}=\sum_{x=0}^{c} \int_{0}^{\tau} f_{T}(t) P_{X}\left(x \mid n_{s} p\right) d t \ldots(8)$

Further, in order to build the model of four components, first we must simplify the new formal for $\mathrm{p}(\mathrm{t})$ by the following steps:-

$\operatorname{pr}(T \leq t \mid T \subset \tau)=\frac{\int_{0}^{t} \lambda e^{-\lambda x} d x}{\int_{0}^{\tau} \lambda e^{-\lambda x} d x}=\frac{1-e^{-\lambda t}}{1-e^{-\lambda \tau}} \cdots(9)$

$p r(t \leq T \subset \tau \mid T \subset \tau)=\frac{\int_{t}^{\tau} \lambda e^{-\lambda x} d x}{\int_{0}^{T} \lambda e^{-\lambda x} d x}=\frac{e^{-\lambda t}-e^{-\lambda x}}{1-e^{-\lambda t}}$

Therefore:-

$p(t)=\frac{e^{-\lambda t}\left(p_{1}-p_{0}\right)+\left(p_{0}-p_{1} e^{-\lambda \eta}\right)}{\left(1-e^{-\lambda \tau}\right)}$
Now: the total function of expected cost consists of:-

$T=A_{1}+A_{2}+A_{3}+A_{4}$

These components are:

$\mathrm{A} 1=c_{I_{r}} n$

$\mathrm{A} 2=c_{R}\left(A_{2}^{*}+A_{2}^{* *}\right) \ldots(12)$

$A_{2}^{*}=$ expected number of rejected units during inspection and is defined by:-

$A_{2}^{*}=E\left(z \mid f_{b}\right) p\left(f_{b}\right)+E\left(z \mid f_{t}\right) p\left(f_{t}\right) p\left(\bar{f}_{b}\right)+E\left(z \mid \bar{f}_{b} \bar{f}_{d}\right) p\left(z \mid \bar{f}_{b} \bar{f}_{d}\right)$

Since we have

$E\left(z \mid f_{b}\right)=\mathrm{n} p_{1} \quad$ and $\quad p\left(f_{b}\right)=B$

Then we get

$$
\begin{aligned}
& \qquad A_{2}^{*}=n p_{1} B+(1-B) \int_{0}^{t} n p(t) f_{T}(t) d t+(1-B) n p_{0}\left(1-\int_{0}^{t} f_{T}(t) d t\right) \\
& \int_{0}^{\pi} p_{X}\left(x \mid n_{s} p(t)\right) f_{T}(t) d t=\int_{0}^{\pi} c_{x}^{n}(p(t))^{x}(1-p(t))^{n-x} \lambda e^{-\lambda t} d t \\
& \text { Since } \\
& \frac{d p(t)}{d t}=\lambda e^{-\lambda t}\left(p_{0}-p_{1}\right) /\left(1-e^{-\lambda t}\right) \\
& \text { And } \\
& I B_{u}\left(\alpha_{i} \beta\right)=\frac{1}{B\left(\alpha_{s} \beta\right)} \int_{0}^{u} z^{\alpha-1}(1-z)^{\beta-1} d z \text { o. (19) }
\end{aligned}
$$

Which is incomplete Beta function, then:-

$$
\begin{aligned}
& \int_{0}^{\tau} p_{X}\left(x \mid n_{s} p(t)\right) f_{T}(t) d t=\left(\frac{1-e^{-\lambda t}}{p_{1}-p_{0}}\right) c_{x}^{n} B(x+1, n-x+1) \\
& \int_{p_{0}}^{p_{1}[p(t)][1-p(t)]^{n-x}} d p(t) \\
& =\left(\frac{1-e^{-2 \pi}}{p_{1}-p_{0}}\right) C_{x}^{n} B(x+1, n-x+1)\left[I B_{p 1}(x+1, n-x+1)\right. \\
& \left.-I B_{p 0}(x+1, n-x+1)\right]_{n}(20)
\end{aligned}
$$

Where

$$
B\left(\alpha_{i} \beta\right)=\frac{\Gamma(\alpha) \Gamma(\beta)}{\Gamma(\alpha+\beta)}
$$

Since we have $(x, n)$ are positive integer , so we can express the incomplete Beta function in terms of Binomial theorem as :-

$$
I B_{p 0}\left(\alpha_{s} \beta\right)=\sum_{i=\alpha}^{n} C_{i}^{n} p_{0}^{i}\left(1-p_{0}\right)^{n-i x}
$$

With

$n=\alpha+\beta-1$

$I B_{p 1}\left(\alpha_{s} \beta\right)=\sum_{i=\alpha}^{n} C_{i}^{n} p_{1}^{i}\left(1-p_{1}\right)^{n-i}$

$I B_{p 0}(x+1, n-x+1)=\sum_{i=x+1}^{n+1} C_{i}^{n+1} p_{0}^{i}\left(1-p_{0}\right)^{n+1-i}$

$I B_{p 1}(x+1, n-x+1)=\sum_{i=x+1}^{n+1} C_{i}^{n+1} p_{1}^{i}\left(1-p_{1}\right)^{n+1-i}$

After simplification we obtain:-

$I B_{p 0}(x+1, n-x+1)=E\left(x+1, n+1, p_{0}\right)$

$I B_{p 1}(x+1, n-x+1)=E\left(x+1, n+1, p_{1}\right)$ 
Therefore

$\int_{0}^{T} p_{X}(x \mid n, p(t)) f_{T}(t) d t=\frac{1-e^{-2 r}}{\left(p_{1}-p_{0}\right)(n+1)}\left[E\left(x+1, n+1, p_{1}\right)-E\left(x+1, n+1, p_{0}\right)\right](22)$

By same method, another computation can used to solve the integral:

$$
\begin{aligned}
& \int_{0}^{t} p(t) p_{X}\left(x \mid n_{s} p(t)\right) f_{T}(t) d t=s^{* *} \\
& \left.s^{* *}=\frac{1-e^{-j t}(x+1)}{\left(p_{1}-p_{0}\right)(n+1)(n+2)}\left[E\left(x+2, n+2 p_{1}\right)-E\left(x+2, n+2 p_{0}\right)\right]\right]_{n(23)}
\end{aligned}
$$

And continue this approach until finding $\left(c^{*}\right)$ which make (w) minimum.

3) Now fix $\left(c^{*}\right)$ and initial value of $(\tau)$ and change (n) by $(\mp S n)$ until you obtain the optimal value $\left(n^{2}\right)$ at which $(w)$ is minimum.

4) The chosen values of $\left(n^{*}{ }^{*} c^{*}\right)$ are considered constant, and then change $(\tau)$ by $(\bar{\Psi} S \tau)$ until the value of $\left(\tau^{*}\right)$ obtained.

5) Repeat the steps $(2,3,4)$ using $\left(\boldsymbol{n}^{*}{ }_{\mathrm{o}} \boldsymbol{c}^{*}{ }^{*} \tau^{*}\right)$ as initial values, if the value of $(w)$ is improved then continue otherwise stop iteration

Until the value of $(w)$ is converges in two successive iterations.

Application of proposed Model

The following data are recorded for certain product production rate (16818 units/day) and production rate per hour (700 unit/hour) .the loss due to stopping of production line because of increasing percentage of defectives from $\left(p_{0}\right.$ to $\left.p_{1}\right)$ is (288 unit /hour)

With

$\left(\alpha_{0}=0.05, \beta_{1}=0.10, p_{0}=0.004, p_{1}=0.05\right)$

$\left(C_{A}=C_{2}=\$ 0.742 /\right.$ unit $)$

( $C_{R}=C_{1}=\$ 0.260 /$ unit $)$

( $C_{I}=C_{0}=\$ 0.009 /$ unit $)$

$\left(C_{F}=C_{3}=\$ 288 /\right.$ unit $)$

The total enumeration procedure is applied for proposed model

And we find the first optimal values are ( $n=290{ }^{*} c=14, \tau=6.5$ hour $)$

Is the best set which total enumeration begin at it, where the value of

$c=1(1) 290$

$n=78(10) \ldots 4550$

$\tau=0.5(0.5) \ldots 24$

So the optimal parameter of sample plan necessary to test the lot

$N=\psi \tau$

According to proposed model are

$n^{*}=235$ units

$c^{*}=6$ units
$T^{*}=3.5$ hours

$w=\$ 324.54$

Expected total cost due to proposed model

Also the method of multivariate search technique and total enumeration was applied for Schmidt-Taylor model and we find the parameters are

$n_{1}^{*}=400$ units

$c_{1}^{*}=7$ units

$T_{1}^{*}=8.5$ hours

$w 1=\$ 386.287$

Therefore the efficiency of proposed Model is

$$
\begin{aligned}
e\left(n_{1}, c_{1}, \tau_{1}\right)=\frac{k_{0}\left(n_{0}, c_{0} \tau_{0}\right)}{k_{1}\left(n_{1}, c_{1}, \tau_{1}\right)} \\
=\frac{324.54}{386.287}=0.84015=84 \%
\end{aligned}
$$

This means that sum of cost and sampling inspection and loss due to wrong decisions can be reduced by $(\mathbf{1 6 \%})$ for using proposed model ,as compared with Schmidt -Taylor

Model.

Building Model of Expectation total cost of quality control

In this section, we shall define another notations as

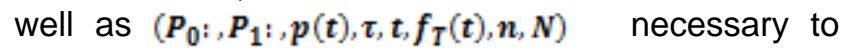
build the model, these are

$\psi$ : production rate (units) is constants $(N=\psi \tau)$

$C_{I^{1}}$ cost inspection per unit

$C_{R^{:}}$cost of rejection good unit due to

wrong decision

$C_{A^{:}}$cost of accepting defective unit

$C_{F}:$ cost of stopping production line

$P(d)$ : probability that process system fail

during production of lot $N$

$f(b)$ : probability of failure in production unit which cause to increase percentage of defective

from $P_{0}$ to $P_{1}$, $\left(P_{1}>P_{0}\right)$

$f(t)$ : probability of failure in production line

during time $\mathrm{t}$, were $(t<\tau)$,

$t(t<\tau)$ through production of lot $N$.

$f(d)$ : probability of failure of production line during some times of producing alot $N$.

$\theta_{i}$ probability that the production system in case of failure after ith inspection.

$P\left(A \mid f_{b}\right)=$ probabilityof accepting alot when the failure occurs at the begining of production a lot $N$ $P\left(A \mid f_{t}\right)=$

probability of accepting alot "when the failure occurs

during any point $t$ during the production lot $N$. 
$P\left(A / f_{d}\right)=$

probability of accepting alot "when the failure occurs

during any point at time of production a lot $N$.

$\boldsymbol{P}\left(A \mid \bar{f}_{b} \bar{f}_{d}\right)$

$=$

probability of accepting a lot when the failure doesnot

happen until finishing the lot $N$

$P_{X}(x \mid n, p)=$ probability that asam

quality $p$ contians $x$ defectives unites

$B=$ probability (in case of stationary )that the productio

in case of failure after any inspection operation.

This means that the new lot begins in production line with $\boldsymbol{P}_{1}$ percentage of defective, and the quantity (B) is computed as follows:-

$B=\lim _{j \rightarrow \infty} \theta_{j}$

Were

Then

$A_{2}^{*}=n p_{1} B+n(1-B)\left[\left(p_{0}-p_{1} e^{-\lambda t}\right)+\frac{1}{2}\left(p_{1}-p_{0}\right) \frac{1-e^{-2 h t}}{1-e^{-\lambda t}}\right]+n p_{0}(1-B) e^{-\lambda t}$

After taking decision of rejection a sample and then rejecting $(N-n)$ units due to increasing number of defectives, then the expected value of rejected unites due to lot rejected $(\mathrm{N})$ is :-

$A_{2}^{* *}=(N-n)$. probability of lot rejection $=$

$(N-n)\left[p\left(\bar{A} \mid f_{b}\right) p\left(f_{b}\right)+p\left(\bar{f}_{b}\right) \int_{0}^{\pi} p\left(\bar{A} \mid f_{t}\right) f_{T}(t) d t+p\left(\bar{A} \mid \bar{f}_{b} \bar{f}_{d}\right) p\left(\bar{f}_{b} \bar{f}_{d}\right)\right]$

$A_{2}^{* \prime}=$

$(N-n)\left[1-B \sum_{x=0}^{6} p_{X}\left(x \mid n, p_{1}\right)-\right.$

$(1-B) \sum_{X=0}^{C} \int_{0}^{T} p_{X}\left(x \mid n_{i} p(t)\right) f_{T}(t) d t-\quad(1-B) \sum_{x=0}^{C} \int_{T}^{m} p_{X}\left(x \mid n n_{0}\right) f_{T}(t) d d_{t}$

Now we derive the component (A3)(Expected Accepted cost , which is

$A_{3}=(N-n)$ Expected value of percentage defective units in accepted lot .

From this definition, we have $A_{3}=(N-n)\left[p_{1} p r\left(A \mid f_{b}\right) p\left(f_{b}\right)+p\left(\bar{f}_{b}\right) \int_{0}^{T} p(t) p\left(A \mid f_{t}\right) f_{T}(t) d t+p_{0} p\left(A \mid \bar{f}_{b} \bar{f}_{d}\right) p\left(\bar{f}_{b} \bar{f}_{d}\right)\right]$

Finally the component $\left(A_{4}\right)$ (loss of stopping of production line when the percentage of defective become $\left(p_{1}>p_{0}\right)$.

Let $I=\left\{\begin{array}{cr}1 & \text { when the production line is closed } \\ 0 & \text { o/w }\end{array}\right.$

Then

$\mathrm{E}(I)=1$ probability the line shutdown 0 probability the line is not shutdown
$\boldsymbol{E}(I)=\boldsymbol{p}\left(\bar{A} \mid f_{b}\right) \boldsymbol{p}\left(f_{b}\right)+p\left(\bar{f}_{b}\right) p\left(\bar{A} \mid f_{d}\right) p\left(f_{d}\right)+p\left(\bar{A} \mid \bar{f}_{b} \bar{f}_{d}\right) p\left(\bar{f}_{b} \bar{f}_{d}\right)$

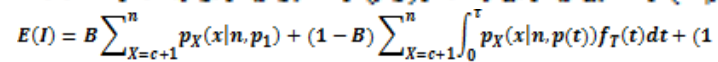

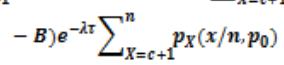

Expected total cost $=C_{I} n+C_{R} A_{2}+C_{A} A_{3}+C_{F} A_{2} \ldots$ (17) Equation (17) represents the general form of total expected quality control for proposed model now we will simplify this form, when the distribution of defective items $(X)$ in sample $(n)$ follow Binomial distribution with $(n, p)$

$f_{s}\left(w_{t} n p\right)=c_{x}^{n} p^{x}(1-p)^{n-x} \quad x=0,1_{v} 2_{s \ldots \ldots} n$

$=0$

o/w

stysiem

1) $p_{X}\left(x \mid n, p_{0}\right)=c_{x}^{n} p_{0}^{x}\left(1-p_{0}\right)^{n-x}$

2) $p_{X}\left(x \mid n_{s} p_{1}\right)=c_{x}^{n} p_{1}^{x}\left(1-p_{1}\right)^{n-x}$

3) $p_{X}\left(x \mid n_{s} p(t)\right)=C_{x}^{n}(p(t))^{x}(1-p(t))^{n-x}$

Another value which must be computed according to Binomial distribution is

$\left(\theta_{1}\right)$ Which the probability of failure after first inspection, by using formula of incomplete Beta, we have:-

$\theta_{1}=\sum_{x=0}^{c} \int_{0}^{t} c_{x}^{n}(p(t))^{x}(1-p(t))^{n-x} \lambda e^{-\lambda t} d t$
$\theta_{1}=\frac{1-e^{-j x}}{\left(p_{1}-p_{0}\right)(n+1)} \sum_{x=0}^{e}\left[E\left(x+1, n+1, p_{1}\right)-E\left(x+1, n+1, p_{0}\right)\right]$

...(24)

The value of $B$ (probability of failure in production system after any inspection) is given by:

$$
\begin{aligned}
& B=\frac{\theta_{1}}{\theta_{1}+1-p\left(A \mid f_{b}\right)}
\end{aligned}
$$

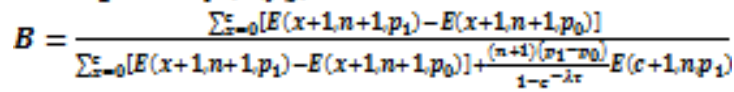

Carry out the application of proposed model to Binomial $(n, p)$, we find :-

$p\left(A \mid f_{b}\right)=\sum_{x=0}^{c} C_{x}^{n} p_{1}^{x}\left(1-p_{1}^{x}\right)^{n-x}=B\left(c, n, p_{1}\right)$

$1-p\left(A \mid f_{b}\right)=\sum_{x=c+1}^{n} c_{x}^{n} p_{1}^{x}\left(1-p_{1}^{x}\right)^{n-x}=B\left(c+1_{s} n_{s} p_{1}\right)$

Putting this result in equation (17) we obtain the expected total cost of quality control for proposed model under Binomial condition

i .e.

$x \sim b(n, p)$

And the time continued until the failure happen follow $\exp (\lambda)$

$f(t)=\lambda e^{-\lambda t}$

The final cost function explained in equation (26)

$r . C=c_{I} n+C_{R}\left\{p_{1} n B+n(1-B)\left[p_{0}-p_{1} e^{-\lambda x}+\frac{1}{2}\left(p_{1}-p_{0}\right) \frac{1-e^{-2 \lambda}}{1-e^{-k x}}+p_{0} e^{-\lambda x}\right]+(N-n)[1-\right.$

$\left.\left.B \beta\left(c, n, p_{1}\right)-(1-B) \theta_{1}-(1-B) e^{-\lambda t} \beta\left(c, n, p_{0}\right)\right]\right\}$ 
$+C_{A}(N-n)\left[p_{1} B \beta\left(c_{s} n_{s} p_{1}\right)+(1-B) S^{* s}+p_{0}(1-B) e^{-\lambda t} \beta\left(c_{s} n_{s} p_{0}\right)\right]$ $+C_{F}\left[B E\left(c+1, n, p_{1}\right)+(1-B)\left(1-\theta_{1}\right)+(1-B) e^{-\lambda t} E\left(c+1, n, p_{0}\right)\right] \cdots(26)$ The mathematical procedure used to solve equation (26) is the multivariate search techniques, which it's computational, can be broken down into the following steps

\section{Calculation Steps}

1) Calculate initial value for total expected cost $\left(T_{x} C\right.$ $=\boldsymbol{w})$, under assumed initial values

$\left(\boldsymbol{c}_{v} \boldsymbol{n}, \boldsymbol{\tau}\right)$, where $(\boldsymbol{c}, \boldsymbol{n})$ are determined according to producers and consumers risk, and chose any value of $(\tau)$

2) Take the initial values of $\left(\boldsymbol{n}_{s} \boldsymbol{c}_{v} \tau\right)$, and fixed $(\boldsymbol{\tau}, \boldsymbol{n})$ and change (c) by $(\mp \delta c)$

\section{SUMMARY AND CONCLUSIONS}

The build model for expected total cost of quality control, consist of four components these are derived directly when the distribution of process is Binomial $(n, p)$.and the Percentage of defective is considered as a function of $(\mathrm{t}) ; \mathrm{P}(\mathrm{t})$, which is a generalization of Schmidt -Taylor model .All notation and explanation are summarized.
A derived equation is used to find the minimum total expected cost of quality control.

And the results are compared with this indicated by Schmidt -Taylor model, the efficiency of two model costs are compared, and we find that the proposed model reduce the loss due to wrong decision of accepting, rejecting and stopping line production ,by (16\%) ,which is considered very good percentage ,especially when the testing is of high cost.

\section{REFERENCES}

1) Guenther, R, W, C. (1977), sampling inspection in statistical quality control chairs Griffin and company $2^{\text {nd }}$.

2) HALD,A.(1981).statistical theory of sampling inspection by attributes academic press INC. London.

3) Schmidt, J. W. and Taylor, R.W (1973) A dual purpose cost based quality control system Technometrics, Vol. 15 No.1 page 151-167.

4) A. Baklizi and A.E.K Elmarsi ,Acceptance sampling plans based on truncated life tests in the Birnbaum sanders model, Ris anal.24(2004)pp-1453-1457 